\title{
News from a Multi-Wavelength Monitoring Campaign on Mrk 421
}

\author{
W. Cui*, for the VERITAS collaboration ${ }^{\dagger}$, M. Blażejowski*, M. Aller**, \\ H. Aller**, H. Teräsranta ${ }^{\ddagger}$, B. Mochejska ${ }^{\S}$, P. Boltwood ${ }^{\mathbb{I}}$, A. Sadun", \\ M. Böttcher ${ }^{\dagger \dagger}$ and A. Reimer ${ }^{\dagger+}$ \\ ${ }^{*}$ Department of Physics, Purdue University, West Lafayette, Indiana, USA \\ ${ }^{\dagger}$ see http://veritas.sao.arizona.edu/VERITAS_members.html for a list of the members \\ ** Department of Astronomy, University of Michigan, Ann Arbor, Michigan, USA \\ ${ }^{\ddagger}$ Metsähovi Radio Observatory, Helsinki University of Technology, Espoo, Finland \\ ${ }^{\S}$ Harvard-Smithsonian Center for Astrophysics, Cambridge, Massachusetts, USA \\ II 1655 Main Street, Stittsville, ON K2S 1N6, Canada \\ " Department of Physics, University of Colorado at Denver, Colorado, USA \\ ${ }^{\dagger}$ Department of Astronomy, Ohio University, Athens, Ohio, USA \\ \#Department of Astronomy, University of Bochum, Bochum, Germany
}

\begin{abstract}
We conducted a daily monitoring campaign on Mrk 421 in 2003 and 2004 with the Whipple $10 \mathrm{~m}$ telescope and the large-area instruments aboard RXTE, simultaneously covering $\mathrm{TeV}$ and X-ray energies. Supporting observations at optical and radio wavelengths were also frequently carried out. Mrk 421 was observed over a wide range of fluxes (with a dynamic range of $\sim 30$ both at $\mathrm{TeV}$ and $\mathrm{X}$-ray energies). The source was relatively quiet in 2003 but became unusually active in 2004, with flares reaching peak fluxes of $\sim 80 \mathrm{mCrab}$ in X-rays and $>3 \mathrm{Crab}$ at $\mathrm{TeV}$ energies! We will describe the multiwavelength campaign and present some preliminary results. We will also discuss the implications of the results on the proposed emission models for $\mathrm{TeV}$ blazars.
\end{abstract}

\section{INTRODUCTION}

Over the past decade, one of the most exciting advances in high energy astrophysics has been the detection of sources at $\mathrm{TeV}$ energies with ground-based $\gamma$-ray facilities (see Weekes 2003 for a recent review). Among the sources detected, blazars are arguably the most intriguing. The emission from a blazar is generally thought to be dominated by radiation from a relativistic jet that is directed roughly along the line of sight (Urry \& Padovani 1995). The spectral energy distribution (SED) of TeV blazars invariably shows two characteristic "humps" in the $v F_{v}$ representation, with one located at X-ray energies and the other at TeV energies (Fossati et al. 1998). There is a general correlation between the two SED peaks. TeV blazars are also known to undergo flaring episodes, both at Xray and $\mathrm{TeV}$ energies. The flares have been observed over a wide range of timescales, from months down to minutes. The observed X-ray flaring hierarchy in Mrk 421 seems to imply a scale-invariant physical origin of the flares (Cui 2004).

A popular class of models associates the X-ray emission from a $\mathrm{TeV}$ blazar with synchrotron radiation from highly relativistic electrons in the jet and the $\mathrm{TeV}$ emission with synchrotron self-Compton (SSC) processes (e.g., Marscher \& Gear 1985; Maraschi 
et al. 1992). The SSC models can, therefore, naturally account for the observed X-ray$\mathrm{TeV}$ correlation. Moreover, they have also enjoyed some success in re-producing the observed SEDs. However, the models face challenges, such as the presence of "orphan TeV flares" (in 1ES 1959+650, Krawczynski et al. 2004; and in Mrk 421, see Fig. 1 in this work). Alternatively, the jet could be energetically dominated by the magnetic field, and it is the synchrotron radiation from highly relativistic protons that might be responsible for the observed TeV gamma rays (Aharonian 2000; Mücke et al. 2003). In such a scenario, the X-rays are thought to be mainly due to synchrotron radiation from co-accelerated electrons in the jet. Although the proton-synchrotron models can also describe the observed SEDs and accomodate the X-ray-TeV correlation, they are being challenged by variability timescales below $\sim 15 \mathrm{~min}$ in TeV blazars. Very recently, it has been proposed that the flares in blazars might be associated with magnetic reconnection events in a magnetically dominated jet (Lyutikov 2003), perhaps similar to solar flares in this regard. The model might offer a natural explanation for the hierarchical flaring phenomenon in Mrk 421, in analogy to solar flares. However, it has not been applied to the data in any quantitative manner.

To make further progress on distinguishing emission models proposed for $\mathrm{TeV}$ blazars, we believe that simultaneous or contemporaneous data are critically needed over a wide range of fluxes, especially in the crucial $\mathrm{X}$-ray and $\mathrm{TeV}$ bands, for quantifying the SED and its variability of a source and for allowing investigations of such important issues as variability timescales, cross-band correlation, spectral variability and hysteresis, and so on. Such data are severely lacking at present, despite intense observational efforts over the years.

\section{THE MONITORING CAMPAIGN}

We conducted a comprehensive multi-wavelength monitoring campaign on Mrk 421. It is the first known $\mathrm{TeV}$ blazar and remains to be the only one that can be detected nearly all the time at $\mathrm{TeV}$ energies. The campaign started in the late Febuary of 2003 and lasted until the early May of 2004. The effort was strongly focused on the X-ray and $\mathrm{TeV}$ bands. We took a snapshot of Mrk 421 at X-ray energies with the PCA detector on $R X T E$ (with typical exposure times of 2-3 ks) and at TeV energies with the Whipple $10 \mathrm{~m}$ telescope (with comparable exposure times), which were coordinated for each night during a Whipple observing period. Supporting observations were also carried out, but less frequently, at 4.8, 8.0, and $14.5 \mathrm{GHz}$ with the $26 \mathrm{~m}$ UMRAO telescope at the University of Michigan, at 22 and $37 \mathrm{GHz}$ with the $14 \mathrm{~m}$ Metsähovi Radio Telescope at the Helsinki University of Technology, and at optical wavelengths with the $1.2 \mathrm{~m}$ telescope (BVRI photometry) at the Fred Lawrence Whipple Observatory (FLWO) and the $0.4 \mathrm{~m}$ telescope ( $\mathrm{R}$ band only) at the Boltwood Observatory. The Boltwood flux measurements are differential; the results have been normalized to those of the FLWO measurements, thanks to a significant overlap of the two observing programs. We note that the error bars shown for the Boltwood data do not include systematic uncertainties and are thus certainly an under-estimation of the overall measurement error. No optical data are currently available for the Whipple 2002/2003 observing season. 


\section{PRELIMINARY RESULTS}

In this section, we present and discuss some of the results from the observations, which we must stress are still preliminary in nature.

\section{Light Curves}

The X-ray and TeV light curves from the entire campaign are shown in Fig. 1, along with radio and optical ones in the selected bands. Note that most Whipple observations were conducted at small zenith angles $\left(<30^{\circ}\right)$. We found that variations in the zenith angle hardly cause any noticeable effects in the TeV light curves shown. In general, Mrk 421 was relative quiet in 2003 , although it was still seen to vary significantly at X-ray and $\mathrm{TeV}$ energies. The measured $\mathrm{X}$-ray and $\mathrm{TeV}$ count rates are roughly correlated but are clearly not always in step (note the presence of "orphan" TeV flares). The latter is even more obvious in 2004, when the source became unusually active. Many major flaring episodes were observed then. For instance, a giant outburst took place near the end of the campaign, with the source reaching peak fluxes of $\sim 80$ mCrab in X-rays and $>3$ Crabs in the TeV band, respectively. It is worth noting that during the giant outburst the $\mathrm{TeV}$ emission appears to reach the peak much sooner than the X-ray emission. The light curves also show that Mrk 421 varies progressively less towards longer wavelengths.
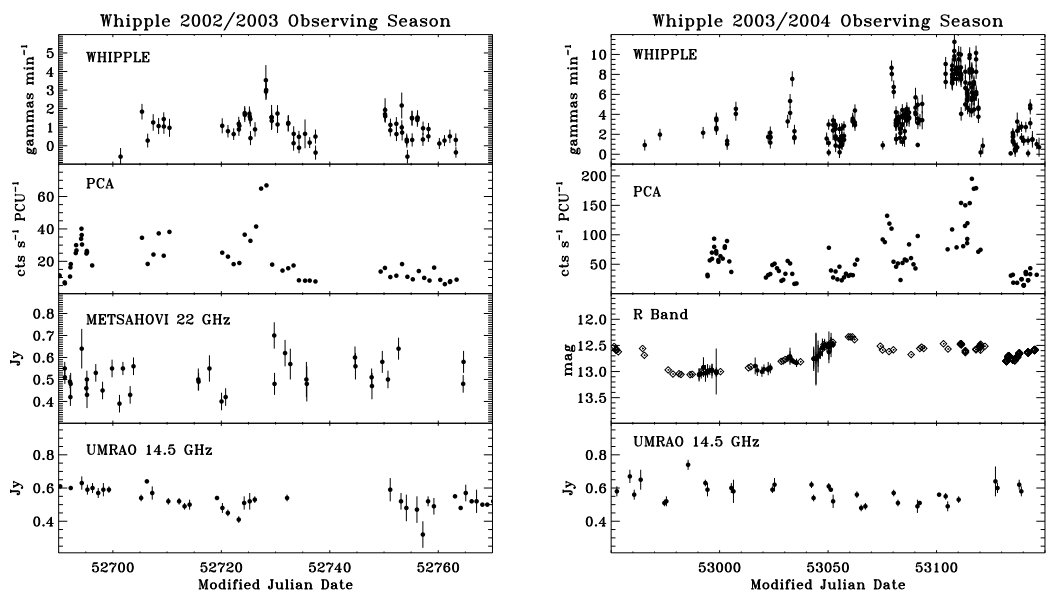

FIGURE 1. Preliminary light curves of Mrk 421. For clarity, the light curves are shown separately for the Whipple 2002/2003 and 2003/2004 observing seasons. For the R-band data, the FLWO points are shown in bullets and the Boltwood points in diamonds. Note that no optical data are available for the 2002/2003 season; the $22 \mathrm{GHz}$ data are shown instead.

\section{X-ray-TeV Correlation}

The correlation between the X-ray and TeV emission can be examined more quantitatively by plotting the count rates against each other, as shown in Fig. 2. We can see 
that the dynamical range of the data collected is quite large, about a factor of 30 in both energy bands, which is important for studying correlative variability of Mrk 421. The correlation is clear from the figure, but it is only a loose one, unlike what one might naively expect based on the SSC scenarios. To be more rigorous, we computed the cross-correlation function between the X-ray and TeV light curves, using the interpolation method, and the results for the 2003/2004 data are shown in Fig. 2. There are two main peaks in the correlation function: one is centered roughly at zero lag and the other at +180 hours. We caution, however, that correlation functions are, in general, prone to systematic effects such as irregular data gaps. An investigation of such effects on our data is under way. If confirmed, the positive lag would imply that on average TeV photons lead X-ray photons, which is opposite to what SSC models predict. Looking at the data more carefully, we noticed that the peak at the positive lag is dominated by data taken during the giant outburst.
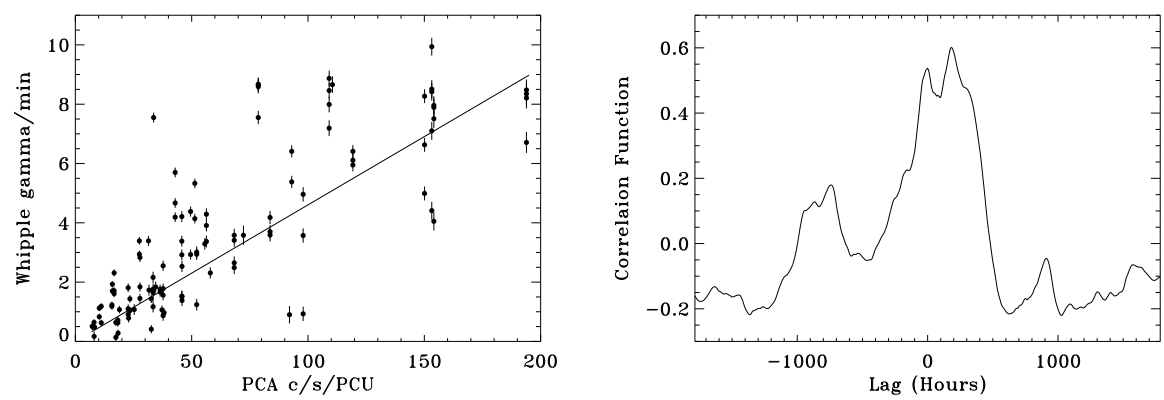

FIGURE 2. (left) Relationship between the X-ray and TeV count rates of Mrk 421. The solid line shows the best linear fit. (right) Cross-correlation function between the X-ray and TeV count rates of Mrk 421 in the Whipple 2003/2004 observing season.

\section{Rapid X-ray and TeV flares}

Zooming onto the X-ray and TeV light curves, we found flares on much shorter timescales. Fig. 3 shows examples of sub-hour X-ray flares. The most rapid X-ray flare lasted only for $\sim 15$ minutes and shows substantial sub-structures, implying variability on even shorter timescales. Since the X-ray emission is almost certainly of synchrotron origin, the timescales associated with such short flares lead to severe constraints on the size of the flaring region, the strength of the magnetic field, and the Doppler factor of the jet bulk motion in a relatively model-independent manner (Cui 2004).

Only on one occasion, a sub-hour X-ray flare was detected during a TeV flare. No counterpart is apparent at $\mathrm{TeV}$ energies, as shown in Fig. 3. The data do not allow comparisons on longer timescales, which shows a serious drawback of the "snapshot" observing strategy. For studying rapid flaring activities of TeV blazars, long, continuous observations are required. This is the trade-off that one must consider, however, given the limited resources available. We detected a number of rapid $\mathrm{TeV}$ flares on timescales of hours, all during the period of the giant outburst. Fig. 4 shows a sample of such flares. Due to observational constraints (note that the coverage was already intensified 

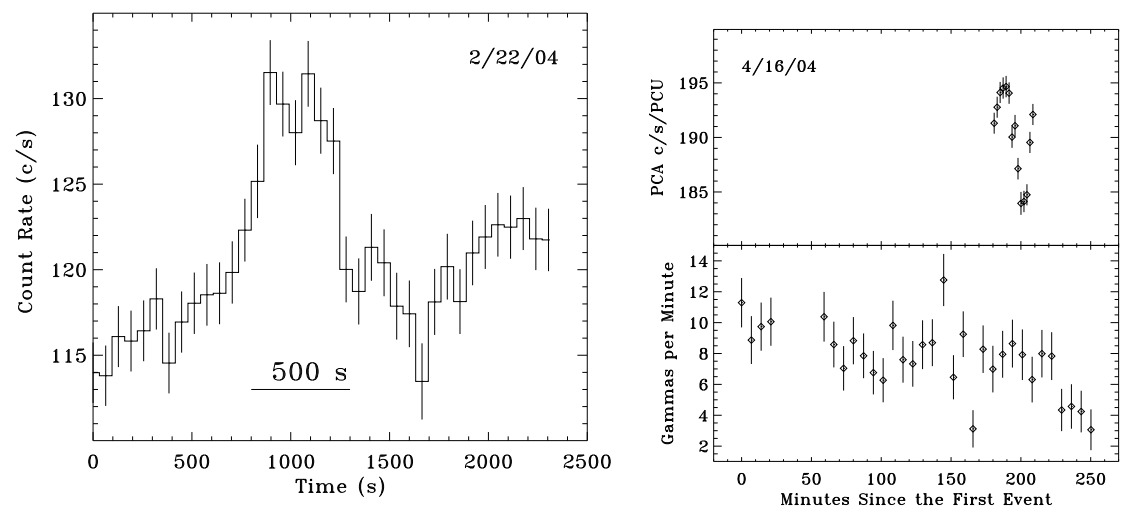

FIGURE 3. (left) Most rapid X-ray flare detected in Mrk 421. Note the sub-structures in the profile of the flare. (right) Sub-hour X-ray flare during a rapid TeV flare (see Fig. 4).

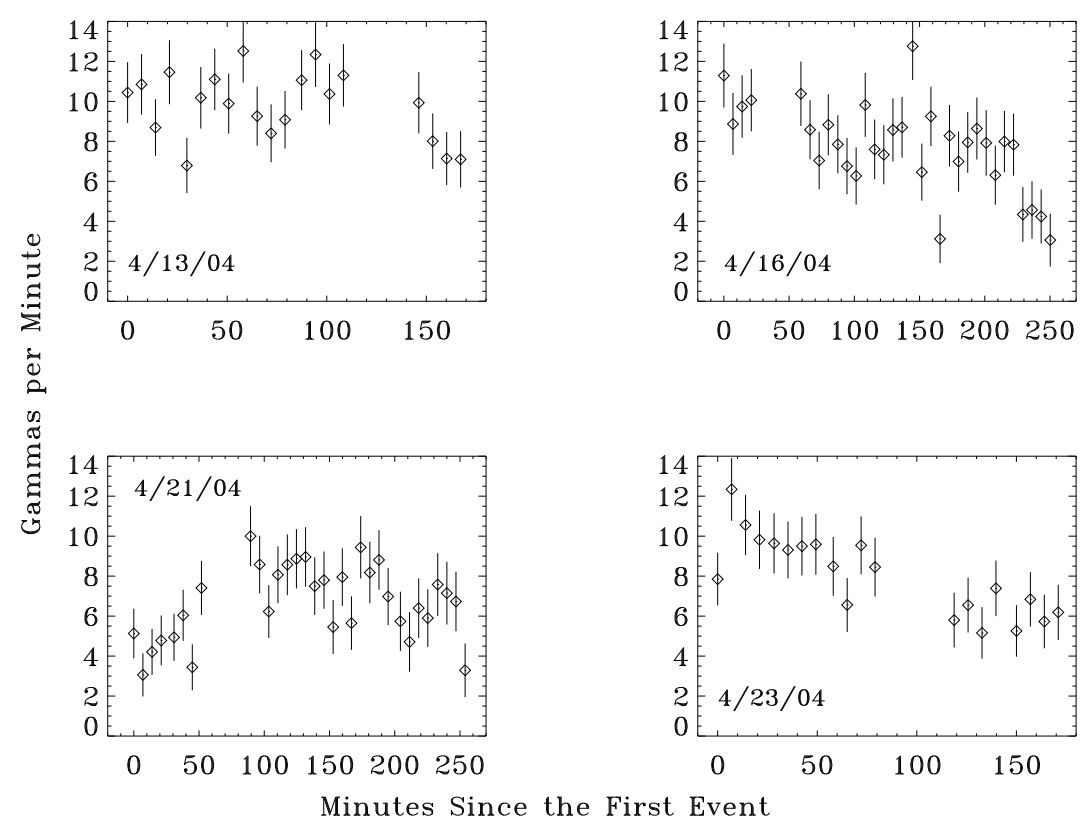

FIGURE 4. Rapid TeV flares in Mrk 421.

at $\mathrm{TeV}$ energies during the period), the flares were only partially covered. Though not statistically significant, weaker flares on shorter timescales appear to be present.

\section{Spectral Energy Distributions}

To examine spectral variability, we divided the observations into 8 groups, based on X-ray count rates of Mrk 421. For each group, we constructed an SED from radio, optical, X-ray, and TeV observations in the group. Note that there usually is a much fewer number of observations at radio and optical wavelengths, while the observations 


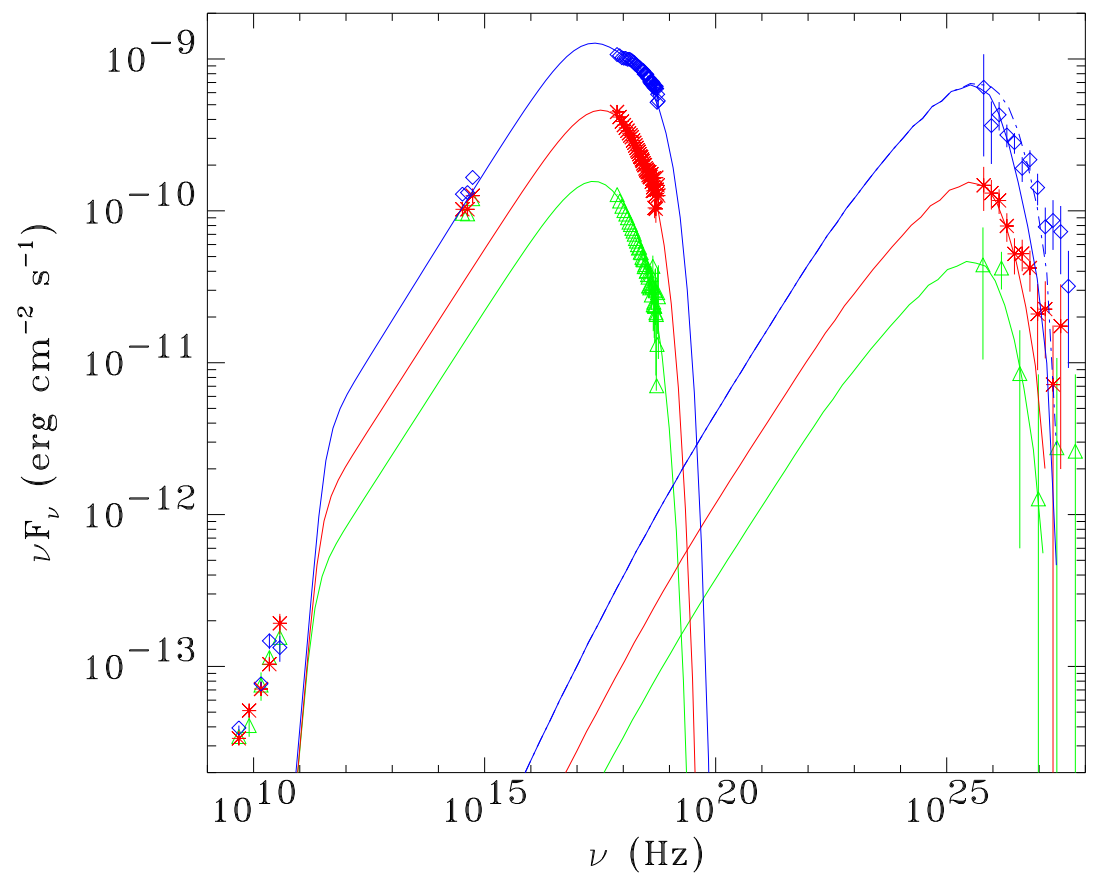

FIGURE 5. Spectral energy distribution of Mrk 421 at different X-ray fluxes. The X-ray flux differs roughly by a factor of 3 between two adjacent SEDs. The solid curves are fits to the data with a onezone SSC model (with contributions from the synchrotron radiation and SSC shown separately). For comparison, the dot-dashed line shows the effect of ignoring absorption by the IR background.

at X-ray and $\mathrm{TeV}$ energies match reasonably well (by design). Since Mrk 421 varies little at longer wavelengths, we believe that the resulted SED should be quite reliable. Fig. 5 shows the SEDs in three of the groups, with each differing from its neighboring group roughly by a factor of 3 in average X-ray count rates.

The SED varies greatly both at X-ray and $\mathrm{TeV}$ energies but little at radio and optical wavelengths. This is hardly surprising in the context of the SSC models, since X-ray and $\mathrm{TeV}$ photons originate from the same population of most energetic electrons in the jet. The synchroton cooling time is much shorter for X-ray emitting electrons than for those contributing to radio or optical emission, which might account for the difference in variability in different bands. The SED is flatter (or harder) at higher fluxes; this is particularly apparent at X-ray energies. This is consistent with results from previous works (e.g., Fossati et al. 2000; Krennrich et al. 2002; Aharonian et al. 2002).

We experimented with a one-zone SSC model ${ }^{1}$ to fit the data, taking into account attenuation of $\mathrm{TeV}$ fluxes by the diffuse infrared background. The results are shown in Fig. 5. The model seems to adequately describe the SEDs at X-ray and TeV energies, although there are still some discrepancies at $\mathrm{TeV}$ energies. On the other hand, it fails badly to account for the measured radio and optical fluxes. This is a generally known problem with one-zone SSC models (e.g., Maraschi et al. 1999; Krawczynski et

1 The description of the code can be found at http://jelley.wustl.edu/multiwave/spectrum/ 
al. 2004), which is hardly surprising since real AGN jets are known to have complicated structures. In modeling, the problem is usually dealt with by invoking multiple populations of electrons (with different energy distributions) in different zones (e.g., Maraschi et al. 1999). The efforts to carry out more sophisticated modeling of the data collected in our campaign are currently in progress.

\section{CONCLUDING REMARKS}

A substantial amount multiwavelength data have been collected on Mrk 421 through our monitoring campaign. The data have allowed us to carry out investigations on variability timescales, cross-band correlation, SED and its flux dependence, and spectral variability and hysteresis across flares. Some preliminary results have been presented in this contribution. They have already begun to shed light on emission mechanisms in $\mathrm{TeV}$ blazars. The study of most rapid flares is hindered by the snapshot observing strategy adopted. It is, therefore, highly desirable to take a complementary approach in the future by observing the source continously for sufficiently long time.

\section{ACKNOWLEDGMENTS}

The VERITAS collaboration is supported by the US Department of Energy, National Science Foundation (NSF), the Smithonian Institution, PPARC (UK), NSERC (Canada), and Science Foundation Ireland. W. Cui and M. Blażejowski also gratefully acknowledge support from NASA. UMRAO is supported in part by funds from the NSF and from the University of Michigan Department of Astronomy.

\section{REFERENCES}

Aharonian, F. 2000, New Astronomy, 5, 377

Aharonian, F., et al. 2002, A\&A, 393, 89

Cui, W. 2004, ApJ, 605, 662

Fossati, G., et al. 1998, MNRAS, 299, 433

Fossati, G., et al. 2000, ApJ, 541, 166

Krawczynski, H., et al. 2004, ApJ, 601, 151

Krennrich. F., et al. 2002, ApJ, 575, L9

Lyutikov, M. 2003, New Astr. Rev. 47, 513

Maraschi, L., Ghisellini, G., \& Celotti, A., 1992, ApJ, 397, L5

Maraschi, L., et al. 1999, 526, L81

Marscher, A. P., \& Gear, W. K., 1985, ApJ, 298, 11

Mücke, A., et al. 2003, APh, 18, 593

Urry, C. M., \& Padovani, P. 1995, PASP, 107, 803

Weekes, T. C. 2003, Proc. 28th ICRC (astro-ph/0312179) 